
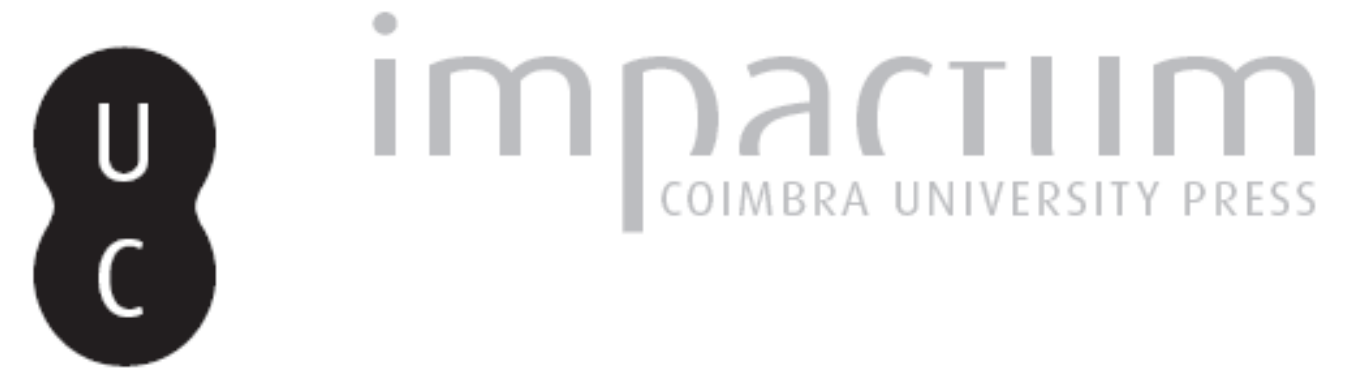

Una sosta "portoghese" in Estremo Oriente: Alberto Moravia a Macao

Autor(es): Petrocchi, Francesca

Publicado por: Imprensa da Universidade de Coimbra

URL persistente: URI:http://hdl.handle.net/10316.2/42452

DOI: $\quad$ DOI:https://doi.org/10.14195/0870-8584_0_8

Accessed : $\quad$ 26-Apr-2023 12:55:30

A navegação consulta e descarregamento dos títulos inseridos nas Bibliotecas Digitais UC Digitalis, UC Pombalina e UC Impactum, pressupõem a aceitação plena e sem reservas dos Termos e Condições de Uso destas Bibliotecas Digitais, disponíveis em https://digitalis.uc.pt/pt-pt/termos.

Conforme exposto nos referidos Termos e Condições de Uso, o descarregamento de títulos de acesso restrito requer uma licença válida de autorização devendo o utilizador aceder ao(s) documento(s) a partir de um endereço de IP da instituição detentora da supramencionada licença.

Ao utilizador é apenas permitido o descarregamento para uso pessoal, pelo que o emprego do(s) título(s) descarregado(s) para outro fim, designadamente comercial, carece de autorização do respetivo autor ou editor da obra.

Na medida em que todas as obras da UC Digitalis se encontram protegidas pelo Código do Direito de Autor e Direitos Conexos e demais legislação aplicável, toda a cópia, parcial ou total, deste documento, nos casos em que é legalmente admitida, deverá conter ou fazer-se acompanhar por este aviso. 


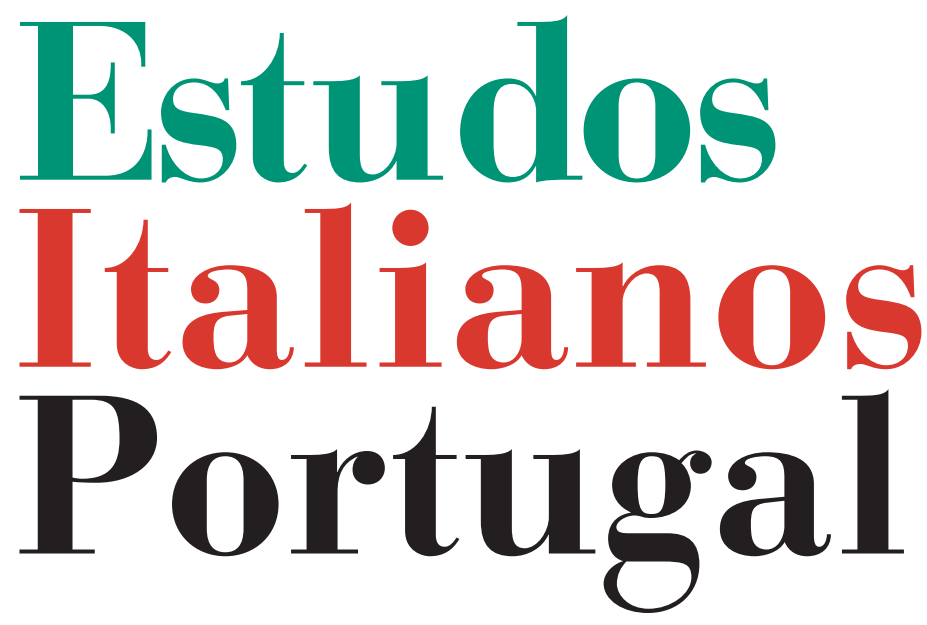

Instituto

Italiano

de Cultura

de Lisboa

Nova Série

$\mathrm{N}^{\mathbf{0}} \mathbf{0}$ 


\section{UNA SOSTA "PORTOGHESE" \\ IN ESTREMO ORIENTE: \\ ALBERTO MORAVIA A MACAO}

Francesca Petrocchi

Tra il FebBraio E L'APrile 1937, Alberto Moravia effettuava un lungo viaggio in Cina, come inviato del quotidiano torinese la Gazzetta del Popolo: un desiderio di "fuga" ("Io facevo viaggi per scappare via dall'Italia" dichiarerà ad Alain Elkann ${ }^{1}$ ) spingeva in realtà Moravia a inoltrarsi nell'“altrove" dell'Oriente, dopo aver investigato, nel 1936, durante un soggiorno di otto mesi, la poliedrica e complessa realtà degli Stati Uniti².

L'ottica di analisi e di decifrazione della società americana che sorregge le corrispondenze giornalistiche edite, tra il marzo-aprile 1936 e l'agosto 1937, sulla Gazzetta del Popolo, in Omnibus (ed una, nel 1941, su Oggi) ${ }^{3}$, era stata tipicamente "eurocentrica", tendendo Moravia a descrivere la "nuova

1 A. Elkann e A. Moravia, Vita di Moravia, Milano, Bompiani,1990, p. 89.

2 Moravia fu invitato da Giuseppe Prezzolini alla Casa Italiana della Cultura della Columbia University di New York, allora diretta da Prezzolini, dove svolse alcune conferenze; "C'è qui da vari giorni Moravia che mi riesce molto simpatico. Non è soltanto uno scrittore. E' un uomo di svariati interessi e di grande acume e di rapida osservazione.Ha capito subito cose dell'America che quelli che arrivan dall'Italia metton anni a intendere. Non si lagna troppo del regime razzista, ma dice che non poteva respirare bene, e lo capisco. Con tutto ciò la vita americana non lo abbaglia e non lo attira": è una annotazione da G.Prezzolini, Diario. 1900-1941, Milano, Rusconi, 1978, p. 552.

3 Le corrispondenze giornalistiche legate al soggiorno negli Stati Uniti sono raccolte in A. Moravia, Viaggi. Articoli 1930-1990, a c. e con Introduzione di E. Siciliano, Postfazione di T.Tornitore, Milano, Bompiani,1994, pp. 109-173. 
civiltà"4 statunitense (pur pulsante e vitale), sottolineando diversità e particolarità emergenti dal confronto serrato con il "vecchio" continente.

Lungo il viaggio in Cina tendeva piuttosto ad investigare tracce e risvolti della dominazione europea, cogliendo il "segno", anche graffiante, di una modernità sempre più evidente nel contesto orientale, in un "mondo settecentesco" quale quello della civiltà cinese ${ }^{5}$, che riproponeva intatta, agli occhi del viaggiatore, "una condizione di vita che non esito a chiamare settecentesca".

Visitando, in particolare, Pechino, Moravia registrava nella pagina narrativa di viaggio la viva impressione di aver compiuto un salto all'indietro nei secoli, il "sentimento di non vivere nel presente, bensì in uno dei passati più illustri e civili che ci siano mai stati"7: e rilevava che il fascino, l'“incanto della vita a Pechino" ("un po' amaro e melanconico") consisteva dunque, per il viaggiatore europeo, nel ritrovare intatte memorie di una vita quotidiana ancora non scalfita dal progresso:

I disastri della Cina vengono in gran parte da questo stacco di secoli che la divide dagli altri paesi. Per questo sono ingiuste le critiche degli stranieri, specialmente degli anglo-sassoni i quali, scambiando una differenza di civiltà per barbarie, parlano spesso della Cina come se fosse uno stato negro dell'Africa. E aggiungiamo che, a parer nostro, quando la Cina sarà quel paese moderno e industriale che gli inglesi vorrebbero che diventasse, sarà certamente più barbara di ora; per convincersene, basta

4 A.Moravia, Gli americani in che modo diventarono americani, in A.M., Viaggi, cit., p. 133.

5 Gli articoli relativi al viaggio in Cina sono raccolti in A.M., Viaggi, cit., pp. 211-383; la citazione è estratta da Aspetti settecenteschi della vita di Pechino, p. 290 .

\footnotetext{
${ }^{6}$ Ivi, p. 289.

7 Ivi, p. 290.
} 
guardare che cosa siano diventati a Sciangai, sola città davvero moderna della Cina, gli antichi gentili costumi e la vecchia saggezza dei cinesi ${ }^{8}$.

Lo scrittore-viaggiatore, animato dall'istanza di comprendere e interpretare il mondo dell" "altrove" andando oltre le apparenze della realtà, era soprattutto attento a cogliere e valutare il complesso problema della "modernizzazione" del variegato e sconfinato mondo cinese: giunto a Canton, che "ha la fama di essere la città più xenofoba e riottosa di tutta la Cina", centro di un intenso traffico commerciale, "ventre", come i quartieri poveri e periferici della Parigi di Zola, della Cina del Sud in quanto "immensa città" ${ }^{10}$ popolata da una massa di miserabili, Moravia tracciava una istantanea descrizione del contraddittorio tessuto socio-economico cinese:

A Canton siamo a tre ore di treno da Hong-Kong; tre ore di treno dalla città europea; dalle grandi banche di granito, dai doks ordinati; dagli alberghi cosmopoliti, dalle caserme dei soldati inglesi, dalle villette dei plutocrati, dai dancings fastosi; eppure è come se si fosse migliaia di chilometri dentro la Cina e lontani dal mare. Perché è tale la condizione cinese: pochi centri progrediti e falsamente europei sulla costa, presidi di civiltà occidentale snaturata e commerciale, porti sicuri per i traffici transoceanici; e poi a pochi chilometri dalla costa città e città ancora feudali, artigianesche, fanatiche, miserabili, incredibilmente pittoresche e per l'europeo talvolta addirittura pericolose. Da Hong-Kong a Canton c'è un salto di parecchi secoli ${ }^{11}$.

Realtà urbane e sociali rimaste ferme nel tempo, e parvenze di una modernità di marca europea "false" , "snaturate"

8 Ibid.

${ }^{9}$ A. Moravia, Canton ventre del Sud, in ivi, p. 240.

${ }^{10}$ Ivi, p. 236.

${ }^{11}$ Ivi, p. 241. 
in quanto innestate forzosamente, e per ragioni espansionistiche, commerciali tipiche dell'aggressivo colonialismo dei "plutocrati", in un contesto bloccato se non al feudalesimo quanto meno al livello di una civiltà pre-industriale, contadina e artigiana: la Cina investigata e descritta da Moravia, attraverso una prosa di viaggio lucidissima e controllata sin nel dettaglio, senza cedimenti al passo lirico o ad un esotismo di maniera (pur invitante dato il fascino del topos "orientale") si proponeva quale sintomatico "campione" delle contraddizioni insite nell'ampio e secolare processo di colonizzazione europea .

Che allo sguardo ed alla sensibilità analitica di Moravia appare concretarsi, nel contesto cinese e sullo sfondo di quello europeo all'altezza del 1937, soprattutto nelle forme di una "modernità" non-integrata, sovrapposta artificiosamente: di una modernità, quale "progresso" attivo nella direzione dell'industrializzazione e del commercio, non raggiunta o conquistata attraverso un lento processo di sviluppo coinvolgente l'intera nazione cinese, ma imposta dall'esterno e soprattutto in quelle zone costiere primi baluardi delle conquiste territoriali operate dagli europei.

Nella descrizione di Hong Kong, fornita in apertura delle corrispondenze giornalistiche, l'attenzione di Moravia era rivolta a registrare - non senza accenti critici - i segni evidenti della dominazione inglese:

in quella chiatta che lentamente mi portava verso la riva cinese ritrovavo la solita aria inglese, quella tenace patina di rispettabilità insipida, di ottimismo in sordina, di commerciale efficienza che i britannici ovunque si stabiliscano stendono anche sopra le realtà più ribelli ed esotiche. [...] Appena sceso a terra questa impressione di trovarmi non in Cina ma in Inghilterra trovò una conferma nell'aspetto della città europea ${ }^{12}$.

12 A. Moravia,Hong-Kong.Una miseria millenaria amministrata con parsimonia britannica, in ivi, p. 223. 
Ma l'impatto con l'uomo trainante il "ricsciò" su cui saliva per effettuare un giro di visita nella città, rivelava, oltre le tranquille, severe apparenze dell'architettura urbana moderna in stile anglosassone, il dramma della povertà, del degrado morale imposto alla popolazione cinese, "questa degradazione dell'uomo che disputa il suo posto alla bestia"13.

Orchestrato sul ritmo del confronto tra le due diverse identità (il mondo cinese rimasto fermo nei secoli, eppur vivo nei costumi atavici e gentili, nelle antiche credenze religiose, nello splendore vigoroso dell'architettura orientale ${ }^{14}$; e quella europea, esportata, trapiantata, e sostanzialmente scontestuata), il racconto di viaggio moraviano trovava una misura narrativa inconsueta rispetto all' andamento complessivo delle corrispondenze, in Un giorno a Macao, edito il 12 maggio 1937, ed in effetti secondo articolo pubblicato sulla Gazzetta del Popolo legato all'itinerario esplorativo della Cina.

L'attenta ricostruzione delle tappe del viaggio elaborata da Tornitore nella Postfazione alla raccolta Viaggi, precisa che Moravia giunse a Macao quando aveva già effettuato gran parte del viaggio, essendosi fermato a Sciangai, Nanchino, Suciao, Pechino, Suan-Hawa-phu, Kalgan, Pechino, per un nuovo ritorno a Sciangai, da dove, imbarcatosi in nave, raggiunse ancora Hong Kong e da lì Macao ${ }^{15}$.

Come accade di frequente nelle corrispondenze giornalistiche di viaggio, l'ordine di pubblicazione degli articoli non collima precisamente con l'ordine di "scrittura" degli stessi: probabilmente la posizione strategica assunta dal racconto della tappa a Macao nell'intero sviluppo delle corrispondenze, posizione che non rispetta lo svolgimento reale del viaggio, è del tutto casuale e forse dovuta a scelte effettuate dalla redazione della Gazzetta del Popolo.

13 Ivi, p. 224.

14 Cfr. in particolare A.Moravia, Il Tempio del Cielo, in ivi, pp. 334-38.

15 Cfr. T.Tornitore, Postfazione, in ivi, p. 1812. 
Eppure, nel racconto legato alla visita di Macao si riversavano riflessioni ed emozioni soggettive efficacemente contrastanti rispetto a quelle registrate nel già citato primo tassello edito delle corrispondenze "cinesi", Hong-Kong. Una miseria millenaria amministrata con parsimonia britannica, costruito sull'alternanza di descrizioni della duplice realtà dell'isola sottoposta al dominio inglese: l'urbanistica "moderna" della zona degli uffici e dei negozi, ed i quartieri cinesi della povertà; figure umane in miseria e lo spaccato brulicante del porto; la "gran nave bianca" che lo attende, e l'umile, "vecchissima" giunca con a bordo la famigliola di piccoli mercanti cinesi .

Macao appariva invece a Moravia, all'opposto, non dissimile da una città "di provincia, non troppo antica, i cui monumenti risalgano tutti, poniamo, alla fine del ' 600 "'16, dal tratto tipicamente europeo, anzi prezioso e raro "esempio" di "provinciale e romita tranquillità", "forse unico" in tutto l'Estremo Oriente ${ }^{17}$; e lo scrittore-viaggiatore investigava le ragioni storiche di quella che gli appariva quasi una miracolosa sopravvivenza della civiltà latina e mediterranea in quell'angolo remoto della Cina:

Il destino ha voluto che nonostante l'espansione trionfante della colonizzazione nordeuropea, il Portogallo conservasse in suo potere questa isoletta; e che il declino del traffico attirato a Hong-Kong impedisse ai portoghesi di introdurre nella loro città tutte quelle innovazioni di carattere inevitabilmente nordamericano che ne avrebbero certamente snaturato l'aspetto. Così Macao è rimasta quello che probabilmente era qualche secolo fa; e al viaggiatore non dispiace di respirarvi la medesima aria che nel Mediterraneo; aria di civiltà latina miracolosamente sopravissuta alla marea anglosassone.

\footnotetext{
16 A. Moravia, Un giorno a Macao, in ivi, p. 229.

17 Ivi, p. 230.
} 
Passaggio che conferma la diagnosi critica dei risultati apportati dal colonialismo inglese, in larga misura in linea con gli orientamenti della polemica "antiplutocratica" e antibritannica che caratterizzava in quegli anni la politica colonialista e "imperialista" di regime: Macao era dunque vista quale topos oppositivo rispetto a quello di Hong Kong, luogo di conservazione, di sopravvivenza dei "segni" della civiltà mediterranea, che attiravano lo sguardo attento del viaggiatore:

Piccolo porto di barche peschereccie, un tempo gran porto per le caravelle minuscole. Un molo si stende da una parte piegandosi come un braccio ed ha una lanterna in cima; un altro molo va dritto fino a cento metri dalla riva, tra queste due barriere, dondolano sull'acqua oleosa poche giunche, qualche motoscafo, un paio di vaporetti ${ }^{18}$.

"Piccolo" porto tranquillo ed a misura d'uomo, contrastante dunque con quello ben più vasto inserito nella grande baia di Hong Kong, brulicante di grandi piroscafi stranieri, di ferry-boat, di luci e cartelloni pubblicitari: l'arrivo a Macao, sembrava essere, per Moravia, l'approdo in un luogo conosciuto ed in una realtà familiare, consueta; con pochi tratti dipingeva le vie del quartiere commerciale di Macao sorto in prossimità del bacino portuale, per poi subito soffermarsi nella descrizione di una "piazzetta molto mediterranea, con le case di un piacevole barocco policromo e un palazzetto quasi nobile con la scritta: Senado" ${ }^{19}$. Così come estesa e particolareggiata era la descrizione dell'architettura e del profilo urbanistico dell'antico centro cittadino:

Girando per Macao quel che colpisce subito è l'architettura delle case. Un barocco lusitano, leggero piacevole e falso. Ogni casa è dipinta di un colore diverso, per lo più tinte chiare che

18 Ivi, p. 231.

19 Ibid. 
sanno di erbe e di fiori e di frutta: giallo limone, azzurro pervinca, rosa fragola, verde pisello, viola, violetta. In fila, in questo sole crudo e umido, con i ciuffi troppo luminosi della vegetazione tropicale che traboccano dai muri nelle vie deserte, hanno una festosità irreale e parodistica di fondali teatrali. Da quei balconcini rugginosi si aspetta che si sporga qualche donna in costume $^{20}$.

La vitale presenza dei caratteri tipici del barocco lusitano in quello spazio d'Oriente sembrava a Moravia quasi irreale; certa "falsità" dell'atmosfera non era tanto dovuta all'insistente profusione del decoro "stucchevole", tardo barocco ("archi, colonne, capitelli, cornici"), "pasticceria architettonica" - come definita dallo scrittore - che pur era di "sollievo" ammirare "dopo la tetraggine del granito e della rispettabilità di Hong- Kong"21, quanto piuttosto all'incunearsi di quegli elementi artistici e architettonici in un contesto "ambientale" tipicamente tropicale, tanto difforme e lontano rispetto al luogo ove quello stile, ridondante ed artificioso, aveva tratto le sue origini storiche ed artistiche.

Moravia restava colpito dall'intenso fascino, dal tratto decadente, quasi "crepuscolare" emanato dalle antiche architetture di Macao ("Provincia, storia, decadenza, dolcezza del sole sulle pietre consunte, silenzio, tutte cose rare e introvabili in Estremo Oriente"), rivelatosi in un "altrove" quale quello della Cina "dove ogni giorno di più prevalgono l'attività e i costumi nordamericani"22. Ma al suo sguardo attento, la città appariva uno spazio in cui il passato si addensava in simulacri della memoria storica, in rovine, come la chiesa di San Paolo, distrutta da un incendio e della quale restava intatta la sola facciata, questa, invece, di "un barocco

\footnotetext{
${ }^{20}$ Ivi, p. 232.

21 Ibid.

22 Ibid.
} 
severo quasi cinquecentesco". La pagina moraviana si caricava di "cose" ormai morte, ma simboli dell'intreccio tra "sacro" e "profano", tra passato e presente:

C'è la statua della Vergine con il braccio alzato, c'è a ricordo dei traffici di Macao una nave della forma di una galiotta; c'è uno scheletro digrignante, vecchio ammonimento cristiano. Ma attraverso le finestre e il portale si spalanca il cielo azzurro; di modo che tutti quei simboli e quelle effigi hanno la inutilità dei vecchi ninnoli disposti sopra i piani di una mensola ${ }^{23}$.

Il racconto sembra aprirsi ad una ripresa dell'apparato simbolico caratteristico del crepuscolarismo: i "vecchi ninnoli" inutili, effigi di una storia caduca, stagliati melanconicamente entro lo scenario tropicale di sfondo che occhieggiava dietro i vuoti della facciata della chiesa ("il cielo tropicale e gli alberi rigogliosi e umidi" ${ }^{24}$ ); Macao era dunque per Moravia "tutta come questa chiesa: una facciata costruita in pietra e coperta di sculture", topos in cui "si misura la vanità di certe rapaci e fruttuose colonizzazioni: le pietre se le rimangiano il sole e la tumultuosa natura, il sangue puro e violento dei dominatori rifluisce sempre più torpido nelle vene dei meticci fino a scomparire diluito nella marea della prolificità cinese" 25 .

L'impatto con il "reliquiario" di un antico splendore, della potenza dei dominatori, dell'orgogliosa utopia di una civiltà che aveva inteso insediarsi entro una natura esotica e nel mondo dell' "altrove", spingeva Moravia a ricercare, aggirandosi per l'isola, altri "segni" e "simboli" della storia: di una storia, sottolineava, "che esercita la sua attrazione soprattutto nei luoghi dove una vitalità decaduta e imbelle la fa considerare come la sola cosa degna di attenzione".

23 Ivi, pp. 232-233.

24 Ivi, p. 233.

25 Ibid. 
A Macao, "come a Pechino, come in certe vetuste e morte città mediterranee", continuava Moravia, "la storia parla di continuo con la voce sommessa e agra del rammarico e dell'orgoglio, accompagna come un'ombra il viaggiatore trasognato e pensoso": un richiamo che per lo scrittore si accende in quei luoghi ove la storia assume oramai l'accezione di "memoria", di passato, le cui tracce visibili sono vuoti simulacri in un presente che non conosce più il "tumulto" vitale del progresso civile.

Non toccata dalle deformazioni imposte dall'aggressivo colonialismo di stampo anglosassone, dall'incalzare del progresso industriale e commerciale, Macao era riuscita a conservare, per Moravia, quei pochi tratti dell'antica forza e dell'antico splendore: tuttavia era comunque "ben morta", ed in attesa, se mai, che "la natura e le finalità internazionali" ${ }^{26}$, dunque la forza erosiva dell'ambiente tropicale da un lato e le ragioni inarrestabili del progresso moderno dall'altro, non cancellassero anche i segni del ricordo della colonizzazione portoghese.

Sosta "mediterranea" lungo l'itinerario in Estremo Oriente, l'esplorazione di Macao e delle rovine del forte militare suscitava in Moravia reazioni oscillanti tra la denuncia dei mali del colonialismo che aveva imposto le apparenze di un progresso sostanzialmente "falso", di sfruttamento economico (e già allora anche "turistico") dei territori coloniali ("Il paesaggio è così bello visto di lassù che con ogni probabilità in una Macao ricca e prospera il forte sarebbe stato spianato, la colubrina e le palle relegate in un museo, e un grande albergo vi sarebbe stato costruito" ${ }^{27}$ ) - denuncia che con maggior vigore e consapevolezza della realtà storica Moravia esprimerà negli anni a venire, quando, a partire dagli anni Settanta, avrà modo di visitare e analizzare il mondo post-coloniale,

\footnotetext{
26 Ivi, p. 234.

27 Ibid.
} 
in particolare quello africano ${ }^{28}$ - e melanconiche constatazioni sulla inarrestabile decadenza della memoria storica, quand'essa è simbolo di un "rapace" quanto "vano" potere.

Si è insistito sulle tonalità e l'atmosfera a nostro avviso "crepuscolari" di alcuni passaggi del racconto di viaggio moraviano, insolite, per altro, nel contesto narrativo dello scrittore romano, in quanto appare di qualche interesse analitico confrontare le impressioni (e le descrizioni) legate a Macao con quelle registrate da Guido Gozzano, allorché nel 1912, ebbe modo di fare tappa lungo il suo viaggio in India - intrapreso anche nella speranza di sfuggire alla malattia - in un'altra colonia portoghese, Goa "la Dourada".

In Gozzano il processo di trasposizione nella scrittura del "viaggio reale" appare per altro sottoposto ad una forte incidenza ri-creativa, in quanto la cronologia del viaggio effettivamente intrapreso non risponde alla datazione dei frammenti che compongono l'insieme delle corrispondenze poi edite prevalentemente sulla Stampa tra il 1912 e il 1913 e pubblicate in volume con il suggestivo titolo Verso la cuna del mondo. Lettere dall'India ${ }^{29}$, nel 1917 un anno dopo la morte dell'autore, con prefazione di Giuseppe Antonio Borgese.

In particolare, la "tappa" a Goa, raggiunta a bordo del "Pedrillo" con partenza da Bombay, viene descritta sotto forma diaristica, con una datazione posticcia (14-17 dicembre 1912), quando invece è certo che Gozzano a fine aprile 1912 iniziò il suo viaggio di ritorno verso l'Italia.

Ma al di là di quei dettagli che possono essere elencati tra le trasposizioni fantastiche o ricreative del Gozzano scrittore

${ }^{28}$ Dalle corrispondenze giornalistiche legate ai frequenti viaggi effettuati in Africa, Moravia estrarrà A quale tribù appartieni?, Milano, Bompiani, 1972; Lettere dal Sahara, Milano, Bompiani, 1983; Passeggiate africane, Milano, Bompiani, 1987; ma si v. anche La rivoluzione culturale in Cina, Milano, Bompiani, 1968.

${ }^{29}$ Tra le varie edizioni, qui si cita da G.Gozzano, Verso la cuna del mondo. Lettere dall'India, Roma, Il Melograno, 1980. 
di viaggio, le pagine dedicate a Goa "simbolo" della colonizzazione portoghese offrono interessanti spunti di comparazione con quelle moraviane incentrate su Macao. Entro l'itinerario esplorativo dell'India, la visita a Goa rappresentava per Gozzano una fuga dall'India del presente (ove coesistevano la realtà antica e fascinosa di una civiltà millenaria e quella del "moderno" ed efficiente colonialismo britannico), verso l'India del "sogno"30; di un sogno avventuroso coltivato sin dalla giovinezza, quando sull'atlante geografico ricercava Goa ove risiedeva un missionario, fratello del suo compagno di banco.

Il primo contatto con la città, raggiunta dopo alcuni giorni di navigazione in un Oceano ricco di fascino esotico, recava il segno del trauma, del contrasto tra "sogno" e "realtà": "Da due ore m'aggiro per la più strana, la più triste delle città morte" (41). La colonia portoghese appariva a Gozzano, come accadrà a Moravia viaggiatore a Macao, una città "che fu", dalle sembianze familiari, consuete, ma ormai spettrali:

La nostra malinconia ritrova invece a Goa lo spettro di cose nostre: conventi, palazzi, chiese del Cinquecento e del Seicento: una vasta città che ricorda a volte una via di Roma barocca o una piazza dell'Umbria: una città che fu suntuosa e ricca, sorta per l'imposizione della croce e della spada ${ }^{31}$.

Graffiante, per Gozzano, il contrasto tra le superstiti architetture del barocco europeo e l'ambiente tropicale, l' "implacabile", dirompente e distruttivo "clima della foresta": "Per le cose come per gli uomini il tropico è deleterio; e sotto questo cielo di fiamma e d'uragano i secoli contano millenni". La corruzione, il disfacimento delle memorie della storia

${ }^{30}$ Cfr. N.Orengo, Presentazione, in ivi, p. 6.

31 G.Gozzano, Goa "La Dourada", in ivi, p. 41. 
venivano colti da Gozzano allorché si addentrava nelle strutture architettoniche ormai solo "di facciata", scatole vuote:

Un edificio m'attira, un palazzo del Seicento, imponente, dalle grate panciute, dai balconi a volute aggraziate, recanti al centro, in corsivo, un monogramma o uno stemma padronale [...] Il cortile è circondato da un doppio loggiato barocco, a colonne spirali; ma il loggiato è crollato per una buona metà e s'apre sopra la campagna selvaggia. Seguo il portico a caso, entro nella vasta dimora. Oimé! Vedo il soffitto; e, attraverso il soffitto, larghe chiazze azzurre: il cielo del tropico. [...] il palazzo non è che una scatola, una topaja deserta, che serve da magazzino per le noci di $\operatorname{cocco}^{32}$.

E dunque il racconto finiva per soffermarsi sulla descrizione di "cose" morte: "Strade interminabili, alternate di palazzi cadenti, vuoti come teschi, di verzura sopravanzante alti muri massicci”.

La vegetazione tropicale ha inghiottito e ricoperto le reliquie di un antico splendore: quello della Goa "regina d'Oriente, orgoglio dei figli di Luso"33, ora ambiente spettrale, carico di simboli in disfacimento che invitava Gozzano ad una inquieta e melanconica riflessione, esemplare entro le costanti della poetica gozzaniana, ove "l'allontanamento dal presente e il rifugio nel mito della memoria" 34 , storica e individuale, acquistano i contorni di una via di fuga dalla realtà contingente, ma solo apparente e momentanea occasione di felicità:

Ancora una volta tocco l'ultimo limite della delusione, sconto la curiosità morbosa di vedere troppo vicina la realtà delle pietre

32 Ivi, p. 42.

${ }^{33}$ Ivi, p. 43.

${ }^{34}$ C. Ghelli, Introduzione a Gozzano e i crepuscolari, a c. di C.Ghelli, Milano, Garzanti, 1983, p. XXVII. 
morte, di voler constatare che le cose magnificate dalla storia, dall'arte, cantate dai poeti, non sono più, non saranno mai più, sono come se non siano state mai.

Il contatto dei due scrittori-viaggiatori con le città-simbolo del colonialismo portoghese nelle terre dell'Oriente suscitava impressioni e reazioni improntate a registrare l'inarrestabile decadenza della potenza e della civiltà europea quando trapiantate nell" "altrove" esotico e selvaggio:

La malinconia della città morta è tutta nel contrasto di questo medioevo europeo, di questo passato nostro, sepolto sotto un cielo d'esilio, in una terra selvaggia ${ }^{35}$.

Di una modernità stridente e provinciale appariva a Gozzano Pandjim, la "Nova Citade" di Goa, che "sembra una città di provincia dei tempi andati, una capitale di qualche Repubblica dell'America Centrale, sul finire del secolo XVIII": il racconto della "sosta portoghese", vissuta quale "giornata malinconica tra le più malinconiche del mio pellegrinaggio" 36 indiano, si chiudeva nel ricorso alla citazione letteraria, ad un sonetto scritto da De Heredia "per la patria lontana", quasi una preghiera proferita da Gozzano "sulla tomba della città defunta"37.

Così, al trionfale "accademico poema di Camoens" celebrante le conquiste di Vasco da Gama, Gozzano opponeva la "Morne Ville, jadis reine des Océans": riferimento alla memoria storica della tradizione letteraria, passata e più recente, che può essere segnalato anche nel racconto della sosta "portoghese" a Macao di Moravia. Che, appena sceso a terra, andava alla ricerca del museo di Camoens, spinto, come affermerà più avanti, dalla sua irresistibile "curiosità"

${ }^{35}$ G.Gozzano, op. cit., p. 44.

${ }^{36}$ Ivi, p. 47.

${ }^{37}$ Ibid. 
di storia: e si aggirava entro un palazzetto (ove la storia era ormai erosa dal tempo), affollato di tombe incassate "tra le lastre del pavimento", corroso da "grandi macchie di umidità", con la "fontana asciutta e ripiena di calcinacci" nel cortile interno, pieno di "vecchie masserizie sgangherate e vetusti e anneriti pezzi di scultura" che "marciscono negli angoli erbosi":

Nel cortile il silenzio è completo, qualche gallina becca tra gli interstizi del lastrico erboso, il sole picchia con forza su tutti quegli intonachi e quelle pietre verdi di licheni e inzuppate di umidità $^{38}$.

Forse coincidenza dovuta al peso che la realtà esterna andava esercitando sulla sensibilità del viaggiatore, ma la descrizione di Moravia si appuntava a tratteggiare uno spazio in disfacimento tipicamente crepuscolare, un topos emergente in numerosi testi poetici del crepuscolarismo, ove le memorie architettoniche di un antico splendore e nobiltà presenti in ville abbandonate, chiuse, in parchi e giardini, convivono con i "segni", del tutto prosaici, del presente, rovesciando la "poetica dello spazio fissata da Pascoli e soprattutto da D’Annunzio"39 e, insieme, i grandi miti dell'arte borghese.

Per Moravia, l'incontro con le testimonianze del passato di Macao fungerà da ulteriore stimolo alla sua diagnosi pessimistica del grande fenomeno storico del colonialismo, della "vanità di certe rapaci e fruttuose colonizzazioni", rovesciando contestualmente la diffusa retorica di propaganda "imperialista" allora in voga in Italia.

Se il racconto della visita a Goa di Gozzano si apriva e si chiudeva nel nome di Camoens, riferimento, del resto, quasi obbligato per un letterato, analogamente questo processo di

\footnotetext{
38 A. Moravia, Un giorno a Macao, cit., p. 231.

39 C. Ghelli, op. cit., p. XXIX.
} 
citazione si ritrova nel racconto moraviano, che apertosi sul tema della ricerca del Museo, si chiudeva con la descrizione delle "grotte di Camoens".

Spazio celebrativo e "museale", con i giardini attorno alla "grotta" festosi e animati di vita, dai "vialetti puliti", le aiuole "smaltate di bei fiori colorati, gli zampilli d'acqua friggenti", vitalità che contrastava con l'atmosfera di "morte" prima riscontrata nella città: e la statua di Camoens, "dagli occhi tristi e pensosi", restava pur sempre l'ultimo frammento di una lunga storia, ancor vitale tuttavia, quasi che la poesia fosse l'unica realtà storica in grado di restare intatta nel tempo. 